\title{
Keynote: Engineering Challenges of New Business Models in Software
}

\author{
Anthony Finkelstein \\ Computer Science, University College London \\ London, UK
}

The software engineering research agenda has largely emerged from experience of software development in the context of established software business models. Thus, principally bespoke software development or more rarely 'product' software development - unitary software delivered under a product licensing agreement to a market. The reshaping of the software business through the introduction of new ways of 'delivering' software with associated business models must necessarily lead us to reconsider the software engineering agenda. Thus applications offered 'as a service', 'apps' with associated 'channels', 'appliances', fine-grain software services and so on each have associated with them unique engineering challenges which need to be addressed.

In this talk I will examine the prospects of each of these approaches, review the associated challenges and set them in the context of broader research directions for software engineering. I will suggest that not only will the content of software engineering research need to change but that the way in which software engineering research is conducted will need to change also. I will introduce the idea, drawn from biomedicine of the 'translational pipeline' and explain how such a pipeline can be implemented. I will describe particular experience with the development of an 'application as a service' offering and highlight some contrasts between that experience and that of developing software within a more conventional product business model. 